\title{
Construction and validation of a scale of assessment of self-care behaviors with arteriovenous fistula in hemodialysis
}

\author{
Clemente Neves SOUSA, ${ }^{1,2,3}$ João Luís Alves APÓSTOLO, ${ }^{4}$ \\ Maria Henriqueta Jesus Silva FICUEIREDO,' Vanessa Filipa Ferreira DIAS, ,,6 \\ Paulo TELES, ${ }^{7}$ Maria Manuela MARTINS ${ }^{1}$ \\ 'Escola Superior Enfermagem do Porto (Nursing College of Porto), Portugal; 'ICBAS-Oporto University, \\ Porto, Portugal; ' ${ }^{3}$ Diaverum Portugal; ${ }^{4}$ Health Sciences, Area of Nursing, Escola Superior Enfermagem \\ Coimbra, Coimbra, Portugal; ${ }^{5}$ ICS-University Católica Porto, Porto, Portugal; ${ }^{6}$ USF St. André de Canidelo, \\ V N Gaia, Portugal; ' School of Economics and LIAAD-INESC Porto LA, Porto University, Porto, Portugal
}

\begin{abstract}
Several guidelines recommend the importance of educating the patient about the care of vascular access. Nurses have a key role in promoting the development of self-care behaviors by providing the necessary knowledge to patients, so that they develop the necessary skills to take care of the arteriovenous fistula (AVF). This article describes the process of building a scale of assessment of self-care behaviors with arteriovenous fistula in hemodialysis (ASBHD-AVF). This is a cross-sectional study in which the development, construction, and validation process followed the directions of the authors Streiner and Norman. This is a convenience sample, sequential, and nonprobabilistic constituted by 218 patients. The study was conducted in two stages during 2012-2014. The first phase corresponds to the scale construction process, 64 patients participated, while the second corresponds to the evaluation of metric properties and 154 patients participated. The principal component analysis revealed a two-factor structure, with factorial weights between 0.805 and 0.511 and between 0.700 and 0.369 , respectively, explaining $39.12 \%$ of the total variance of the responses. The Cronbach's alpha of the subscale management of signs and symptoms is 0.797 and from the subscale prevention of complications is 0.722 . The ASBHD-AVF revealed properties that allow its use to assess the self-care behaviors in the maintenance and conservation of the AVF.
\end{abstract}

Key words: Arteriovenous fistula, hemodialysis, self-care, nursing care, scale

\section{INTRODUCTION}

In the last years, the prevalence of chronic kidney disease (CKD) has increased about $20 \%$ to $25 \%$ worldwide, ${ }^{1}$ exerting more pressure on health systems. It is estimated that the number of new cases of CKD will exceed 450,000

Correspondence to: C. N. Sousa, PhD, Escola Superior de Enfermagem do Porto, Rua Dr. António Bernardino de Almeida, Porto 4200-072, Portugal. E-mail:

clementesousa@esenf.pt in the United States alone in 2030 and that 2 million people will need dialysis or kidney transplantation. ${ }^{2}$ Thus, a large number of patients will need vascular access.

Vascular access is essential to the achievement of treatment and is one of the main causes of mobilizing economic resources, hospitalizations, and morbidity in dialysis patients. The United States has spent about \$1 billion for the maintenance of vascular access in patients, translating into approximately 6700 to 7900 dollars per patient per year, ${ }^{3}$ which means $17 \%$ of the resources available for the treatment of hemodialysis (HD). ${ }^{4}$ The Vascular 
Access Guidelines ${ }^{5}$ and the Europerian Guidelines recommend the importance of educating patients about the care of vascular access. Nurses have a key role in promoting the development of self-care behavior by providing the necessary knowledge to patients so that they develop the necessary skills to take care of the arteriovenous fistula (AVF).$^{6-10}$ The teachings should be directed to the specific care in the maintenance and functioning of the AVF, namely those associated with the prevention of infection and thrombosis, the aseptic precautions prior to puncture, and the care to treat hematomas. ${ }^{8,9}$

Pagels et al. ${ }^{11}$ have shown that information conveyed by the nurse related to vascular access improve self-care of the patient with the access. The information must be provided in a structured and systematic manner to facilitate the acquisition of these behaviors. It is important that the person with AVF understands that the development of self-care behaviors is a precaution that they can develop, with the aim of reconciling their lifestyle to the current condition of health. Self-care behaviors are actions that patients perform to access their arm in order to verify, maintain, and preserve the functionality of the AVF.

In this context, adapting the definition of Orem, ${ }^{12}$ selfcare is defined as the behaviors performed by the person intentionally for the maintenance of health and wellbeing, which aim to promote the patency of the AVF. The self-care behaviors are learned and acquired during the individual growth process through interpersonal relationships and contexts. ${ }^{12}$ The patient making those self-care behaviors is developing a set of cognitive and perceptual skills to identify situations that compromise the AVF. This situation can reduce costs associated with maintenance of vascular access and the patient can detect early the possible complications of the AVF. Self-care is a key measure for improving the quality of health care that does not depend on technology. ${ }^{13}$

Sousa et al. ${ }^{9}$ conducted a review study verifying the lack of tools to assess the self-care behaviors with the AVF performed by patients. Thus, an instrument that identifies not only if some patients are able to take care of their AVF, but also the proportion of patients who are not able to do it, allows us to identify how many patients need to be educated.

Moreover, the need for an instrument that can be used to assess the patient's self-care as well as educational programs for the development of self-care behaviors in patients with AVF is perceptible. This article describes the process of design and development of the scale of assessment of self-care behaviors with arteriovenous fistula in hemodialysis (ASBHD-AVF) and the results of the process demonstrating the metric qualities of the instrument.

\section{METHODS}

This is a cross-sectional study in which the development, construction, and validation of the process followed the directions of authors Streiner and Norman. ${ }^{14}$ The study was conducted in two stages (Figure 1) during 20122014 with the aim of constructing a scale allowing the evaluation of self-care behaviors of patients with CKD in dialysis. The first stage corresponds to the scale construction process, while the second corresponds to the evaluation of metric properties.

\section{Construction process}

Self-care behavior was defined as all behaviors performed by the person intentionally to maintain the health or wellbeing ${ }^{12}$ directed to the verification and maintenance of the functionality of the AVF in HD. We considered each behavior of self-care as an item. The construction of the instrument began with the compilation of a set of self-care behaviors to include in the scale, based on clinical experience and literature review conducted by the researcher in the area of vascular access for HD. ${ }^{9}$ We had a guideline to build a simple scale without redundancy, allowing good acceptance/understanding by patients.

\section{Content validity}

Content validity was ensured by two panels of experts. The first panel consisted of nephrology nurses $(n=4)$, experienced in the evaluation of vascular access. The second panel was composed of nephrology nurses $(n=5)$ and nephrologists $(n=3)$, which integrate the consultation team in vascular access, with knowledge of the content to conceptual structure. In each panel, the experts were asked to provide feedback about clarity/conciseness, the similarity with other items, and relevance to the construct of each item. For clarity/conciseness, we sought to know if the item was understandable, perceivable, using simple and unequivocal terms, and without possibility of other interpretations. For similarity to other items, we intended to find out if the item was not confusing and if it does not give the idea of repetition. For relevance to the construct, we intended to know the importance of the item to assess the self-care behavior. The experts reviewed the items held against the above-mentioned criteria using the scale of agreement: $-1=$ strongly disagree; $0=$ neither disagree/neither agree; $1=$ agree. If the score was -1 or 0 , the experts were asked to suggest changes that seemed more relevant or to rewrite the item.

This process resulted in a version of 31 items that were arranged in a Likert-type scale of 5 points, ranging from 1 
FirstPhase

Construction of ASBHD-AVF

Second Phase

Test the reliability and validation of ASBHD-AVF
Theoretical foundations
- Review of literature
- Definition of items

Content validity

Expert panel

- First panel: $n=4$, nephrology nurses

- Second panel: $n=8$, nephrology nurses $(n=5)$ and nephrologists $(n=3)$

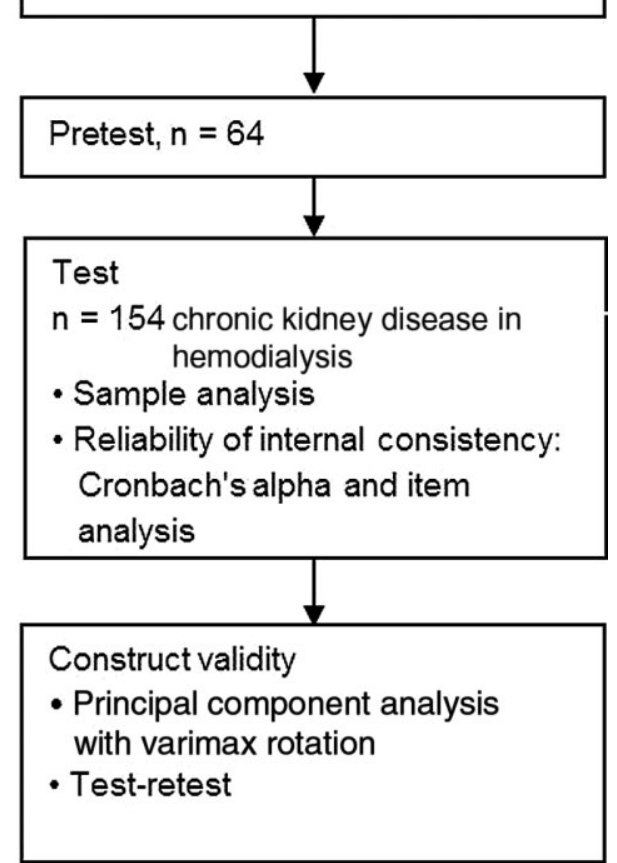

Figure 1 Development process of the scale of assessment of self-care behaviors with arteriovenous fistula in hemodialysis (ASBHD-AVF).

"never develop this self-care" to 5 "always develop this self-care."

One pretest was performed with the version of 31 items, which contained 10 reverse-scored items, which was applied to the sample of 64 renal patients in HD (ASBHD-AVF-31). The opinion of patients, reanalysis of the content of the items made by the experts, and internal consistency led to the elimination of 11 items and originated a version of 20 items.

Final version

A re-examination of experts and metric analysis showed a version of 16 items, which was designated as ASBHD-
AVF-16. ASBHD-AVF has a minimum of 16 and maximum of 80 points (Figure 2), the higher the score the better the self-care behavior of the person in the maintenance and upkeep of the AVF.

$$
\text { ASBHD-AVF }=\left(\frac{\text { Patient score }-16}{64}\right) \times 100 \%
$$

Figure 2 Formula for calculating the scale of assessment of self-care behaviors with arteriovenous fistula in hemodialysis (ASBHD-AVF)-16. 
Table 1 Sociodemographic characteristics of individuals who constitute the two samples $(n=64$ and $n=154)$

\begin{tabular}{|c|c|c|c|c|}
\hline & \multicolumn{2}{|c|}{ First sample $(n=64)$} & \multicolumn{2}{|c|}{ Second sample $(\mathrm{n}=154)$} \\
\hline & $\mathrm{N}$ & $\%$ & $\mathrm{~N}$ & $\%$ \\
\hline \multicolumn{5}{|l|}{ Gender } \\
\hline Male & 40 & 62.50 & 108 & 70.1 \\
\hline Female & 24 & 37.50 & 46 & 29.9 \\
\hline \multicolumn{5}{|l|}{ Scholarity } \\
\hline 4 years & 39 & 60.94 & 90 & 58.44 \\
\hline 6 years & 4 & 6.25 & 8 & 5.19 \\
\hline 9 years & 12 & 18.75 & 24 & 15.58 \\
\hline $11-12$ years & 5 & 7.81 & 17 & 11.04 \\
\hline Superior & 4 & 6.25 & 15 & 9.74 \\
\hline \multirow[t]{2}{*}{ Age } & \multirow{2}{*}{\multicolumn{2}{|c|}{$\begin{array}{l}\text { Minimum: 21; Maximum: } 86 \\
\text { Mean: } 64.66, \text { SD: } 14.80\end{array}$}} & \multirow{2}{*}{\multicolumn{2}{|c|}{$\begin{array}{l}\text { Minimum: } 21 \text {; Maximum: } 88 \\
\text { Mean: } 63.40, \text { SD: } 14.39\end{array}$}} \\
\hline & & & & \\
\hline 6-Item cognitive impairment test & \multicolumn{2}{|c|}{ Mean: 2.91, SD: 2.36} & \multicolumn{2}{|c|}{ Mean: 2.13, SD: 2.25} \\
\hline
\end{tabular}

\section{Sample}

This study involved 218 patients who had end-stage renal disease who underwent HD in dialysis centers in Northern Portugal. The study was conducted in two samples, the first $(n=64)$ and second $(n=154)$ patients, whose sociodemographic characteristics are presented in Table 1. Inclusion criteria for the sample were as follows: patients older than 18 years, carry HD for more than 6 months with AVF, and with no cognitive impairment. We used the 6-item cognitive impairment test to evaluate the cognitive status of each patient, adapted and validated for Portugal by Paiva and Apóstolo. ${ }^{15}$

Participants were invited to participate in the study while waiting to start HD. This is a convenience sample, sequential, and nonprobabilistic.

\section{Procedures}

The study was approved by the ethics committee of the research unit in health sciences: Opinion 78-03/2012. Patients completed a questionnaire after signing informed consent. The instrument was applied on two occasions to all respondents, with a difference of 4 weeks between applications. For patients who had no limitations in interpreting, the questionnaire was self-completed. Those who showed some limitation had assistance by the researcher.

\section{Statistical analysis}

The analysis was performed using the Statistical Package for the Social Science Software (version 21.0; SPSS Inc., Chicago, IL, USA). We proceeded to the analysis of reliability and fidelity through the internal consistency by Cronbach's alpha and by the test-retest temporal stability.
Construct validity was analyzed through principal components analysis with varimax rotation, suppressing the lower factorial loads to 0.30 . A P value 0.05 was considered statistically significant.

\section{RESULTS}

The literature review enabled to build 31 items that should integrate ASBHD-AVF. The first analysis of content validity held by experts originated that the following eight items were rewritten: number 1 "I wash the fistula arm with soap and water before hemodialysis" which became "I wash the fistula arm with soap and water before entering the hemodialysis room;" number 7 "I apply ointment on the sites of bruising" which became "I apply ointment when hematoma occurs;" number 13 "Feel the thrill in the fistula site at least twice a day" which became "I feel the thrill at the site of the fistula twice a day;" number 16 "I notice signs of infection (warmth, redness and swelling) at the sites of stings" which became "I observe signs of redness and swelling at the puncture sites;" number 17 "I allow the measurement of blood pressure in the fistula arm" which became "I allow blood pressure to be evaluated in the fistula arm;" number 18 "I protect the fistula arm of situations that can cause infection, including scratches, cuts and wounds" which became "I protect the fistula arm from scratches, cuts and wounds;" number 29 "I carry weight with the fistula arm" which became "I carry weights using the fistula arm;" and number 30 "If the fistula site has not thrill I will immediately go to the hospital" that changed to "I immediately go to hospital or a clinic if fistula has not thrill." Any more items were eliminated or included. Reanalysis by the experts resulted in $100 \%$ consensus, being designated ASBHD-AVF-31. 


\section{Analysis of internal consistency}

The ASBHD-AVF-31 was administered to an initial sample of 64 patients and was then subjected to an initial analysis of internal consistency revealing values of Cronbach's alpha coefficient ranging between 0.751 and 0.782 (Table 2). The data on the correlation of the item with the total score of the scale revealed that problematic items were eliminated; the numbers $4,5,8,9,11,17,20,21$, 23,25 , and 28 presented the correlation values between -0.17 and 1.49 and had ambiguous content. A second analysis of internal consistency of the 20 items, which revealed Cronbach's alpha values ranging between 0.801 and 0.820 , which allow considering them much good, was accomplished.
The ASBHD-AVF-20 was administered to the second sample of 154 patients, the internal consistency analysis has shown that two items, number 1 and 12, showed low correlations with the total score of the item (0.49 and 0.95 , respectively), therefore did not meet the criteria set by Streiner and Norman. ${ }^{14}$ The remaining 18 items have shown that Cronbach's alpha varies between 0.756 and 0.794 .

\section{Analysis of construct}

The principal components factor analysis revealed a structure with originally six factors explaining $63.19 \%$ of the cumulative variance. The revealed structure was difficult to understand because of the combination of items or

Table 2 Correlations of items with the total score of scale of assessment of self-care behaviors with arteriovenous fistula in hemodialysis (ASBHD-AVF)-31 ( $\mathrm{n}=64)$

\begin{tabular}{|c|c|c|}
\hline Items of ASBHD-AVF & $\begin{array}{l}\text { Corrected } \\
\text { item-Total } \\
\text { correlation }\end{array}$ & $\begin{array}{l}\text { Cronbach's } \\
\text { alpha if item } \\
\text { was deleted }\end{array}$ \\
\hline 1-I wash the fistula arm with soap and water before entering the hemodialysis room & 0.372 & 0.761 \\
\hline 2-I address the nurse when I have cramps during hemodialysis & 0.607 & 0.751 \\
\hline 3-I do compression of puncture sites with the fingers (hemostasis) & 0.217 & 0.769 \\
\hline 4-I move the fistula arm during hemodialysis & 0.095 & 0.776 \\
\hline 5-I control the amount of fluid I drink at home & 0.145 & 0.774 \\
\hline 6-I address the nurse when I get a headache and chest during hemodialysis & 0.561 & 0.753 \\
\hline 7-I apply ointment when hematoma occurs & 0.450 & 0.757 \\
\hline 8-I do compression of puncture sites using tweezers & 0.020 & 0.777 \\
\hline 9-I remove dressings of the puncture sites at night or the next day to hemodialysis & 0.140 & 0.772 \\
\hline 10-I apply ice in the first 24 hours in the local hematoma & 0.288 & 0.767 \\
\hline 11-I remove crusts from puncture sites of the fistula at home & -0.017 & 0.776 \\
\hline 12-I apply heat on local hematoma after the first 24 hours & 0.337 & 0.764 \\
\hline 13-I feel the thrill at the site of the fistula twice a day & 0.497 & 0.754 \\
\hline 14-I do compression at home at puncture site if bleeding occurs & 0.344 & 0.763 \\
\hline 15-I check every day if the hand of the fistula arm cools & 0.347 & 0.763 \\
\hline 16-I observe signs of redness and swelling at the puncture sites & 0.414 & 0.759 \\
\hline 17-I allow blood pressure to be evaluated in the fistula arm & 0.149 & 0.773 \\
\hline 18-I protect the fistula arm from scratches, cuts and wounds & 0.382 & 0.763 \\
\hline 19-I check every day if the color of the hand from the fistula arm changes & 0.369 & 0.762 \\
\hline 20-I apply ointment in local hematoma in the days of hemodialysis & 0.043 & 0.782 \\
\hline 21-I use tight clothes in the fistula arm & -0.010 & 0.777 \\
\hline 22 - I protect the fistula arm from bumps and shocks & 0.328 & 0.764 \\
\hline 23-I sleep over the fistula arm & -0.007 & 0.779 \\
\hline 24-I allow blood sampling in the fistula arm & 0.281 & 0.768 \\
\hline 25-I shave the hairs on the fistula arm & 0.063 & 0.774 \\
\hline 26-I address the nurse if the hand of the fistula arm start to hurt & 0.439 & 0.760 \\
\hline 27-I avoid getting into places with different temperatures & 0.273 & 0.767 \\
\hline 28-I use bracelets and watches on the fistula arm & 0.023 & 0.777 \\
\hline 29-I carry weights using the fistula arm & 0.193 & 0.770 \\
\hline 30-I immediately go to hospital or a clinic if fistula has not thrill & 0.516 & 0.752 \\
\hline 31-I address nurses if the hand of the fistula arm appear with wounds & 0.464 & 0.758 \\
\hline
\end{tabular}


Scree plot

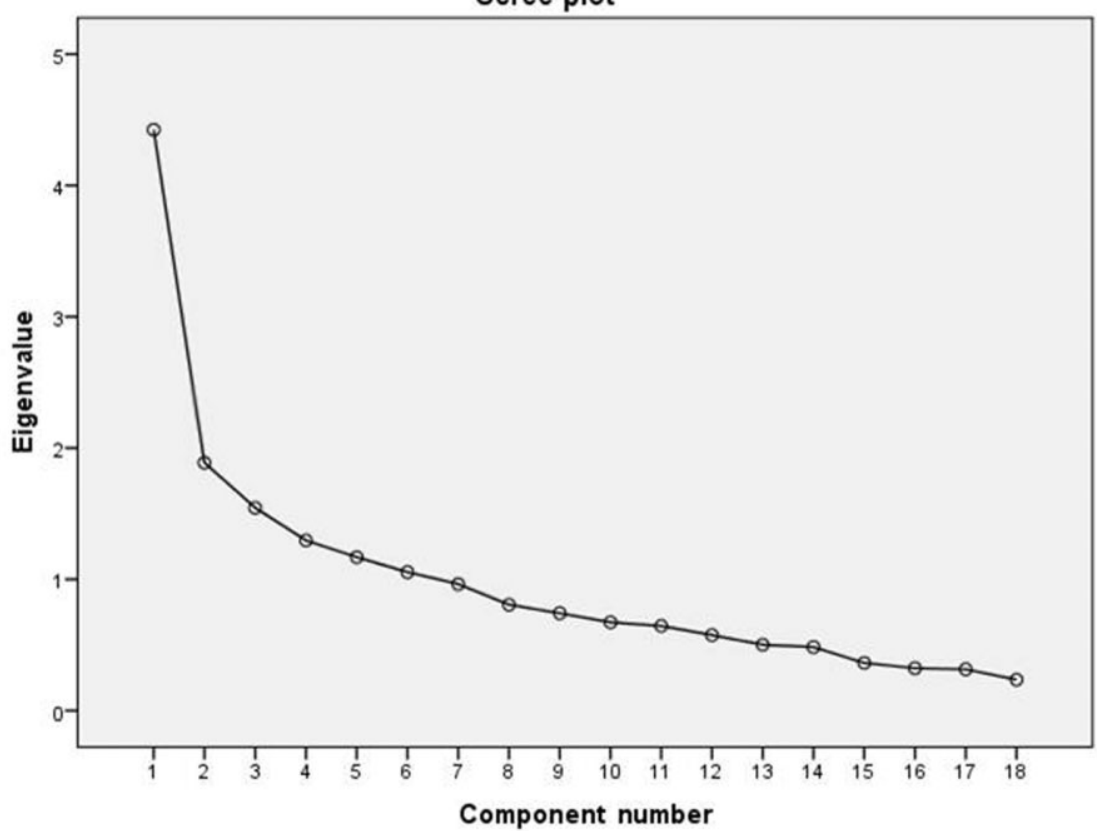

Figure 3 Cattell scree test component factors of scale of assessment of self-care behaviors with arteriovenous fistula in hemodialysis (ASBHD-AVF)-18.

factors, but Cattell Scree test showed a break in the first two factors (Figure 3).

One principal component extraction was performed using an orthogonal varimax rotation, forced to two factors and factorial loads lower than 0.30 were removed. Data analysis suggested the removal of two items (10 and 29) having a load factor lower than 0.30. A structure resulted from two factors that correspond to management of signs and symptoms and prevention of complications that explain $39.12 \%$ of the total variance (Table 3 ). The first factor groups the items belonging to the subscale signs and symptoms managed with factorial weights between 0.805 and 0.511 . The second factor groups the items belonging to the subscale prevention of complications with factorial weights between 0.700 and 0.369 .

\section{Study of reliability}

\section{Internal consistency}

The analysis of internal consistency of the items retained in the factor analysis of the full scale (ASBHD-AVF-16) showed a Cronbach's alpha of 0.797 , varying the values of the corrected correlation of each item with the total scale between 0.286 and 0.519 . The items constituting the subscales management of signs and symptoms and prevention of complications revealed Cronbach's alphas of 0.797 and 0.722 , respectively. Item 24 was reversed to make the score scale.

\section{Temporal stability}

The results of the paired t test between the sum scores obtained in the test and retest (4-week interval) in total scale and the respective dimensions (management of signs and symptoms and prevention of complications) reveal that there are differences in means $(P>0.05)$ and a very strong and significant correlation between 0.91 and 0.96 $(\mathrm{P}<0.05)$ (Table 4). The results of the paired t test indicate good temporal stability of the scale.

In this sample, on average, $82.35 \%$ (minimum $21.88 \%$; maximum 100\%) of the self-care behaviors aimed at keeping and checking the functionality of the AVF were made by patients.

\section{DISCUSSION}

The results show that the ASBHD-AVF-16 is a tool to assess the quality of self-care behaviors in the maintenance and preservation of AVF in HD patients. 
Table 3 Matrix of key components of the scale of assessment of self-care behaviors with arteriovenous fistula in hemodialysis (ASBHD-AVF)-16 forced after varimax rotation for two factors and Cronbach's alpha $(\mathrm{n}=154)$

\begin{tabular}{|c|c|c|c|c|c|}
\hline \multirow[b]{2}{*}{ Items of ASBHD-AVF } & \multirow[b]{2}{*}{ Mean } & \multirow[b]{2}{*}{ SD } & \multicolumn{2}{|c|}{ Factorial load } & \multirow[b]{2}{*}{$\begin{array}{c}\text { Cronbach's } \\
\text { alpha }\end{array}$} \\
\hline & & & $\begin{array}{l}\text { One } \\
\text { factor }\end{array}$ & $\begin{array}{l}\text { Two } \\
\text { factor }\end{array}$ & \\
\hline 26-I address the nurse if the hand of the fistula arm start to hurt & 4.68 & 0.838 & 0.805 & & 0.797 \\
\hline 31-I address nurses if the hand of the fistula arm appear with wounds & 4.74 & 0.877 & 0.804 & & \\
\hline 6-I address the nurse when I get a headache and chest during hemodialysis & 4.56 & 1.009 & 0.760 & & \\
\hline 2-I address the nurse when I have cramps during hemodialysis & 4.56 & 0.956 & 0.729 & & \\
\hline 14-I do compression at home at puncture site if bleeding occurs & 4.60 & 1.070 & 0.518 & & \\
\hline 22-I protect the fistula arm from bumps and shocks & 4.67 & 0.908 & 0.511 & & \\
\hline 15-I check every day if the hand of the fistula arm cools & 4.02 & 1.426 & & 0.700 & 0.722 \\
\hline 13-I feel the thrill at the site of the fistula twice a day & 3.81 & 1.387 & & 0.687 & \\
\hline 30-I immediately go to hospital or a clinic if fistula has not thrill & 4.44 & 1.333 & & 0.672 & \\
\hline 19-I check every day if the color of the hand from the fistula arm changes & 3.38 & 1.697 & & 0.577 & \\
\hline 3-I do compression of puncture sites with the fingers (hemostasis) & 4.79 & 0.749 & & 0.541 & \\
\hline 24-I allow blood sampling in the fistula arm * & 4.91 & 0.516 & & 0.421 & \\
\hline 7-I apply ointment when hematoma occurs & 4.15 & 1.454 & & 0.402 & \\
\hline 18-I protect the fistula arm from scratches, cuts and wounds & 4.74 & 0.739 & 0.315 & 0.383 & \\
\hline 16-I observe signs of redness and swelling at the puncture sites & 3.76 & 1.560 & 0.342 & 0.373 & \\
\hline 27-I avoid getting into places with different temperatures & 2.89 & 1.394 & & 0.369 & \\
\hline
\end{tabular}

Kaiser-Meyer-Olkin: 0.775; chi square approximate-Bartlett's sphericity test 683,767; P 0.000.

* Reversed item

In the process of metric analysis (ASBHD-AVF-31 version), 11 items were eliminated and three items ("I remove crusts from puncture sites of the fistula at home," "I sleep over the fistula arm," and "I use tight clothes in the fistula arm") were eliminated because they had a negative correlation with the factor, which means they have an opposite behavior to that of other items. These self-care behaviors should not be done because the withdrawal of crusts from the puncture sites will enhance the infection, as well as enhance the risk of AVF thrombosis because they can cause distal hypoperfusion. ${ }^{9}$ Patients do not do these behaviors, but they do the other self-care behaviors. We chose to eliminate these three items. Items ("I control the amount of fluid I drink at home", "I do compression of puncture sites using tweezers," "I move the fistula arm

Table 4 Test-retest for equality of the scale and dimensions of scale of assessment of self-care behaviors with arteriovenous fistula in hemodialysis (ASBHD-AVF)

\begin{tabular}{lcc}
\hline Scale and dimensions & $\mathrm{r}$ & $\mathrm{P}$ value \\
\hline Scale ASBHD-AVF & 0.94 & 0.000 \\
Management of signs and symptoms & 0.96 & 0.000 \\
Prevention of complications & 0.91 & 0.000 \\
\hline
\end{tabular}

$r=$ Pearson's correlation; $\mathrm{P}<0.05$. during hemodialysis," "I apply ointment in local hematoma in the days of hemodialysis," "I remove dressings of the puncture sites at night or the next day to hemodialysis," "I allow blood pressure to be evaluated in the fistula arm"' "I use bracelets and watches on the fistula arm"' and "I shave the hairs on the fistula arm") were eliminated because of its ambiguous content; the correlation of the item with the total score is below 0.145 and of low relevance to the instrument as considered by experts.

In the metric analysis (version ASBHD-AVF-20), it was found that two items ("I wash the fistula arm with soap and water before hemodialysis" and "I apply heat on local hematoma after the first 24 hours") had correlations of the item with low total score, so we opted to remove these items because of interference in the Cronbach's alpha. ${ }^{13}$ Items ("I apply ice in the first 24 hours in the local hematoma" and "I carry weights using the fistula arm") were eliminated because they presented low factorial loads, less than $0.30 .^{13}$

For the subscales management of signs and symptoms and prevention of complications, Cronbach's alphas are 0.797 and 0.722 , demonstrating the reliability of the instrument. The subscale management of signs and symptoms refers to a collection of self-care behaviors that the patient recognizes or perceives as changes in the functioning of the AVF. The subscale prevention of 
complications groups items associated with self-care behaviors to prevent or detect AVF complications, including infection, thrombosis, and steal syndrome.

In this sample, on average, over $80 \%$ of self-care behaviors that are intended to the verification and maintenance of functionality AVF were made by patients. However, there are patients that perform these self-care behaviors in a very low percentage $(21.88 \%)$ thus requiring education.

It is intended in future to apply ASBHD-AVF at a new and larger sample to examine the convergent validity and confirmatory factor analysis. The ASBHD-AVF can be used in further research to evaluate the strategies used in programs aimed at developing self-care behaviors in patients with AVF in HD treatment.

\section{CONCLUSION}

The ASBHD-AVF consists of 16 items with a total value of Cronbach's alpha of 0.797 . This is a scale composed by two subscales: management of signs and symptoms and prevention of complications. The first subscale consists of six items with a Cronbach's alpha value of 0.797 and the second subscale consists of 10 items with a Cronbach's alpha value of 0.722 . The ASBHD-AVF revealed properties that allow its use to assess the self-care behaviors of patients on HD in the maintenance and upkeep of the arteriovenous fistula.

\section{ACKNOWLEDGMENTS}

We thank all nurses and doctors that participated in this study, especially MD Ana Ventura and RN Sónia Marques from the Centro de Hemodiálise do Atlântico, Unidade de Gaia; MD Serafim Guimarães and RN Anselmo Madureira from the Caledial, Centro de Hemodiálise de Gaia and MD Mário Oliveira and RN Graciete Martins from the Centrodial, Centro diálise.

Manuscript received July 2014; revised October 2014.

\section{REFERENCES}

1 USRDS. Excerpts From the United States Renal Data System 2008 Annual Data Report: Atlas of Chronic Kidney Disease \& End-Stage Renal Disease in the United States. Am J Kidney Dis. 2009; 53:S81-S90.
2 Schoolwerth AC, Engelgau MM, Hostetter TH, et al. Chronic kidney disease: A public health problem that needs a public health action plan. Prev Chronic Dis. 2006; 3:A57.

3 Steinman TI. Economic issues in dialysis: Influence on dialysis-related complications in the managed-care. In: Lameire N, Mehta RL, eds. Complications of Dialysis. New York, New York, Marcel Dekker. 2000; 737-752.

4 Olmos A, López Pedret J, Piera L. Acceso vascular en hemodiálisis. In: Arias Aljama M, Valderrábano F, eds. Insuficiencia Renal Progresiva. Madrid, Grupo Entheos. 2000; 171-184.

5 NKF-K/DOQI, Kidney Disease Outcomes Quality Initiative. Clinical practice guidelines for vascular access: Update. Am J Kidney Dis. 2006; 48(Suppl 1):S176-S276.

6 Tordoir J, Canaud B, Haage P, et al. EBPG on vascular access. Nephrol Dial Transplant. 2007; 22(Suppl 2):ii88iil17.

7 Sousa CN. Cuidar da Fístula Arteriovenosa: Dos pressupostos teóricos aos contextos das práticas. Master Degree Dissertation presented to the Instituto Ciências Biomédicas Abel Salazar in Oporto University, Portugal; 2009.

8 Sousa CN. Caring for the person arteriovenous fistula: Model for continuous improvement. Rev Port Sau Pub. 2012; 30:11-17.

9 Sousa CN, Apóstolo JL, Figueiredo MH, Martins MM, Dias VF. Interventions to promote self-care of people with arteriovenous fistula. J Clin Nurs. 2013; 23:17961802.

10 Sousa CN, Apóstolo JL, Figueiredo MH, Martins MM, Dias VF. Physical examination: How to examine the arm with arteriovenous fistula. Hemodial Int. 2013; 17:300306.

11 Pagels AA, Wång $\mathrm{M}$, Wengström $\mathrm{Y}$. The impact of a nurse-led clinic on self-care ability, disease-specific knowledge, and home dialysis modality. Nephrol Nurs J. 2008; 35:242-248.

12 Orem DE. Nursing: Concepts of Practice. St. Louis (MO), Mosby; 2001.

13 Horsburgh ME. Self-care of well adult Canadians and adult Canadians with end stage renal disease. Int J Nurs Stud. 1999; 36:443-453.

14 Streiner DL, Norman GR. Health Measurement Scales: A Practical Guide to the Development and Use, 4th edn. New York (NY): Oxford University Press; 2008.

15 Paiva DS, Apóstolo JLA. Cognição e Envelhecimento: Estudo de Adaptação Transcultural e Validação do Six Item Cognitive Impairment Test (6-Cit). Trabalho apresentado no $4^{\circ}$ Colóquio "Envelhecimento, Saúde e Cidadania", Coimbra, Portugal; 2013. 